\title{
THE FORTY-THIRD REGULAR MEETING OF THE SAN FRANCISCO SECTION
}

The forty-third regular meeting of the San Francisco Section of this Society was held in Room 72, Mathematics Building, Stanford University, on Saturday, April 5, 1924, with Professor A. F. Carpenter in the chair. The following twenty-six members of the Society were present.

Alderton, Allardice, Bell, Blichfeldt, Buck, Cajori, A. F. Carpenter, De Cou, Green, Griffin, Growe, Haskell, Hewes, Hoskins, Lehmer, S.H.Levy, McFarland, W. A. Manning, Moreno, F. R. Morris, Noble, T. M. Putnam, Pauline Sperry, Stromquist, A. R. Williams, Wong.

After a discussion of the question as to what form of organization would best serve the interests of mathematics on the Pacific Coast, it was voted that the San Francisco Section continue to have two regular meetings a year, as heretofore, one at Stanford University, and one at the University of California; that a third regular meeting be held annually early in August in the Pacific Northwest; that a fourth regular meeting should be held in Southern California when this should be desirable.

A motion that the Section should not participate in the 1924 meeting of the Pacific Division of the American Association for the Advancement of Science was carried.

Titles and abstracts of the papers read at this meeting follow. The papers by Professors Eells, James, and Milne were read by title.

1. Professor E. T. Bell: Reductions of enumerations in homogeneous forms.

This paper appeared in the July number of this BuLJETIN.

2. Professor E.T. Bell: The class number relations implicit in the Disquisitiones Arithmeticae.

This paper appeared in full in the May-June number of this Bulletin. 
[October,

3. Professor E. T. Bell: On the inversion of arithmetic products.

The Dedekind inversion of a finite product which yields the equation (or congruence) of primitive roots is shown to be a very special case of a general inversion process stated and proved here.

4. Professor E. T. Bell: The numbers of representations of integers in certain ternary quadratic forms.

The number $N\left(n=a x^{2}+b y^{2}+c z^{2}\right)$ of representations of the integer $n>0$ in the indicated form is determined in terms of the binary quadratic class number in each of the nine cases $(a, b, c)=(1,1,2),(1,2,2),(1,2,4),(1,1,4),(1,4,4)$, $(1,2,8),(1,1,8),(1,4,8),(1,8,8)$. Hitherto there have been given only the results for $(1,1,1)$ (Gauss), and incomplete statements for $(1,1,2)$ (Torelli), $(1,2,2)$ (Stieltjes).

5. Professor E. T. Bell: The class number relations implicit in Jacobi's theta formula.

The relations are of a wholly new type involving an arbitrary even function whose arguments are linear functions of the indeterminates representing a fixed integer, whose negative is the argument of the class number functions involved, in certain quaternary quadratic forms.

6. Professor E. T. Bell: Certain products involving the divisors of numbers.

Certain very general identities between infinite products of the type $\Pi\left(1-a x^{b n}\right)^{g(n)}$, in which the $\Pi$ extends to $n=1,2, \ldots, a, b$ are constants, and $g(n)$ is a single valued function of $n$, are obtained and expanded in series. The identities yield many new theorems in partitions, particular cases of which have been given by Jacobi and others.

7. Professor E. T. Bell: Complete class number expansions for the elliptic theta constants of degree 3 .

The nine possible expansions of the type $\vartheta_{\alpha}^{a} \vartheta_{\beta}^{b}(a+b=3$; $\alpha, \beta=0,1,2,3)$ are found by elementary processes. The coefficients are class number functions; the series for $\boldsymbol{\vartheta}_{0}^{2} \boldsymbol{\vartheta}_{3}, \boldsymbol{\vartheta}_{0} \boldsymbol{\vartheta}_{8}^{2}$ have not previously been given. The rest were obtained at greater length by Kronecker and Hermite. 
8. Professor Florian Cajori: Note on our sign of equality.

Professor E. Bortolotti has sent the author a photograph of a page of an undated manuscript now kept in the library of the University of Bologna, written by Pompeo Bolognetti, who before 1568 (possibly before the appearance of Recorde's algebra) used the sign $=$ for equality in the solution of equations.

9. Professor Florian Cajori: History of the use of pictographs and algebraic symbols in elementary geometry.

An account is given of the 250-year struggle between writers friendly and those hostile to the use of symbols in geometry. There still persists a deplorable lack of uniformity in notation.

10. Professor Florian Cajori: Notes on Luca Pacioli's Summa.

In this paper the editions of 1494 and 1523 of the Summa are compared. It is found that Pacioli's use of $\boldsymbol{B}$ as an algebraic symbol has been misinterpreted by certain European and American writers on the history of notations. Pacioli sometimes used a dash to denote equality.

11. Professor Florian Cajori: Despiau's Select Amusements.

Despiau's Select Amusements in Philosophy and Mathematics, brought out at London in 1800 in French and in 1801 in English translation, is a unique book which has escaped the net of nearly all large libraries and of bibliographies of mathematical recreations. It is described in this paper.

12. Professor A. F. Carpenter: Cone-cubic configurations of a ruled surface.

Associated with each line element $g$ of the general ruled surface $S$ there are two cone rays. The points in which these rays cut the flecnode tangents of $S$ drawn at the flecnode points of $g$ are centers of perspective for certain configurations of cone-cubic points and cone-cubic osculating planes. The dual property holds for the planes determined by the cone rays and the flecnode tangents. These perspectivities lead to many interesting theorems which are indicated in this paper. 
[October,

13. Professor W. C. Eells: On a standard definition of the standard deviation.

In this paper the author examines critically numerous definitions of the standard deviation $(\sigma)$. The paper will appear in the Journal of Educational Research.

14. Professor F. L. Griffin: Note on the yield of serial bonds issued at split rates of interest.

To determine the yield on bonds, purchased at any market price, maturing serially in unequal amounts, and carrying two or more different rates of interest, requires the solution of a complicated equation of high degree. The author points out a convenient adaptation of Newton's method in connection with the ordinary bond tables, and discusses some fallacious methods commonly used by investors.

15. Professor Glenn James: On the solution of algebraic equations with rational coefficients.

A generalization of the ordinary factor theorem is used to determine quadratic factors. This paper will appear in the American Mathematical Monthly.

16. Professor W. E. Milne: Note on the flexure of an I-beam.

The problem of determining the stresses in a beam bent by a terminal transverse load can be solved in terms of a function $\varphi(x, y)$, harmonic at all points of the cross section and having assigned values for the normal derivative on the boundary. The problem is easily solved if the boundary of the section is a circle, an ellipse, or a rectangle. The important case of an I-beam may be solved by means of a certain hyperelliptic integral. In this note it is shown how this integral may be evaluated to any desired degree of approximation by means of theta functions. It is further shown that for beams ordinarily used in structural work the constants are very small, so that the series used converges rapidly. The first two terms of the series will ordinarily give results with an error not exceeding onethousandth of one per cent.

\author{
C. A. Noble, \\ Acting Secretary.
}

